\title{
Design Methodology for High Brightness Projectors
}

\author{
Florian Fournier, Student Member, IEEE, and Jannick Rolland, Member, IEEE
}

\begin{abstract}
The low luminance levels of light-emitting diodes (LEDs) compared to arc lamps make it difficult to design high-brightness LED-based projectors. Besides, the specificities of LEDs do not always allow using the same design schemes as with arc lamp-based projection displays. This paper performs a taxonomy of the techniques that can be used to increase the brightness of LED-based projection displays. We show that, in étendue-limited systems, the perceived brightness depends on the system étendue limit, the efficiency of the light engine, and the source luminance. The ability to improve each of these parameters depends on the design constraints. The system étendue limit can be increased at the expense of bulkier, more complex, and more expensive designs. The light engine efficiency can be increased by using free-form shape components adapted to the shapes and the emission patterns of the considered LEDs. The apparent source luminance can be increased at the expense of the flux by either recycling light or restricting the light collection to a smaller étendue with higher average luminance. Luminance can also be increased by using multiple color primaries (spatial multiplexing) or pulsed LEDs (temporal multiplexing). Finally, we review how light recycling can be implemented to convert polarization without increasing étendue.
\end{abstract}

Index Terms-Geometrical optics, light-emitting diode (LED) displays, lighting, liquid-crystal displays, photometry.

\section{INTRODUCTION}

O VER the past decade, improvements in solid-state technologies have enabled the use of light-emitting diodes (LEDs) for new applications, such as general lighting, automotive lighting, and displays. Achieving high brightness displays is always beneficial, as it enables their use for new applications and under less stringent viewing conditions. In projection displays, light needs to flow through a light valve (the microdisplay which creates the image) and a projection optics (which magnifies the microdisplay onto the screen). These constraints on the spatial and angular extent of the beam make it challenging to obtain high brightness LED projectors. Understanding the tradeoffs caused by the étendue conservation law is critical to optimize the performance of the light engine. In this paper, we review the techniques that have been used to maximize the lumen output of projectors and see how they can be applied to LED projectors.

\section{How CAN THE SCREEN BRIGHTNESS BE INCREASED?}

The visual sensation we refer as brightness is linked to the luminance of objects [1]. The higher the luminance of the screen,

Manuscript received May 18, 2007; revised July 10, 2007. This work was supported in part by VDC Display Systems and the Office of Naval Research Virtual Technologies and Environments (VIRTE) program.

The authors are with the CREOL, College of Optics and Photonics, University of Central Florida, Orlando, FL 32816 USA (e-mail: ffournie@creol.ucf.edu).

Digital Object Identifier 10.1109/JDT.2007.907110 the brighter the image will be to the viewer. The luminance of the screen depends on the image size and also on the reflective properties of the screen: a perfect diffuser will scatter the incoming light everywhere, whereas a partially reflective screen will concentrate the light towards the viewer's location. The screen gain $G(\theta)$ describes this capacity of a screen to reflect light in a particular direction $\theta$, relatively to a perfectly diffusing screen. Given these two parameters, image area $A$ and screen gain $G$, the luminance $L$ perceived by the viewer is, within the approximation of a uniformly illuminated screen, given by

$$
L_{\text {screen }}=\frac{G_{\text {screen }} \Phi_{\text {projector }}}{\pi A_{\text {image }}}
$$

where $\Phi$ is the lumen output of the projector. The perceived brightness can therefore be increased by reducing the size of the image, by using high-gain screens, or by increasing the lumen output of the projector.

Étendue is a useful quantity to determine the maximum amount of light that can be collected by an optical system. Étendue quantifies the spatial and angular extent of a light beam. It can be proven that in any optical system, étendue can only increase [2]. This is in fact linked to the second law of thermodynamics. The best we can possibly do is to conserve étendue throughout the light engine. The goal of an efficient light engine is therefore to convert the spatial and angular extent of the light at the source to fit the microdisplay size and the projection lens numerical aperture while keeping the étendue constant. This tradeoff is illustrated on Fig. 1. Accurate étendue calculations are cumbersome, but in the case of a flat emitting surface normal to the optical axis with a uniform beam divergence angle, its expression is given by

$$
E=n^{2} A \cdot \Omega=n^{2} A \pi \sin ^{2} \theta_{1 / 2}
$$

where $n$ is the refractive index of the medium, $A$ the emission area, and $\theta_{1 / 2}$ the half-angle of the emission cone. $\Omega$ is defined as the projected solid angle. For a uniform Lambertian emitter, the luminance of the source $L_{\text {source }}$ is defined as the luminous flux $\Phi_{\text {source }}$ per unit of étendue

$$
L_{\text {source }}=\frac{\Phi_{\text {source }}}{E_{\text {source }}} .
$$

Luminance is often said to be conserved in optical systems. This is known as the luminance conservation law. This is only true if both flux and étendue are conserved. Unfortunately, real systems with passive optical components produce losses, thus decreasing the flux; and aberrations, diffraction and scattering increase the étendue. Luminance, therefore, tends to decrease in optical systems. 

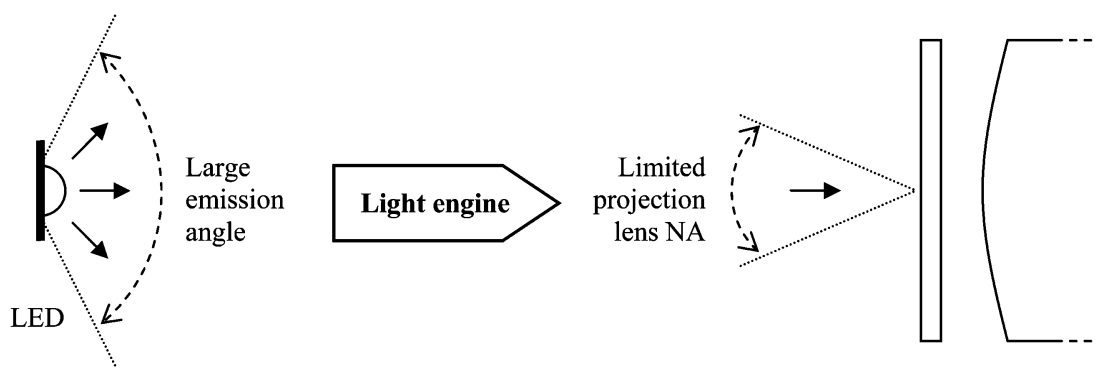

microdisplay

Fig. 1. An efficient light engine collects and shapes the light from the source to fit the microdisplay and the lens while conserving the étendue. The large angular extent of the light at the source is converted to a smaller angle that fits the lens numerical aperture at the expense of a larger area, (area $\times$ projected solid angle is constant)

Any optical system has a limit to the étendue it can collect. If the étendue of the beam is larger than this limit, some light will be cropped by the system. In a projector, the area of the microdisplay and the numerical aperture of the projection lens set stringent limitations on the maximum usable étendue. Given a microdisplay with an area $A$, and a telecentric projection lens with a numerical aperture NA, the étendue limit is given by

$$
E_{\max }=\pi A_{\text {panel }} \mathrm{NA}^{2} .
$$

We can then obtain a first-order estimate of the maximum achievable projector lumen output for an étendue-limited system, i.e., when $E_{\text {source }} \geq E_{\max }$

$$
\Phi_{\text {projector }}=\eta L_{\text {source }} \cdot E_{\max }
$$

where $\eta$ is the efficiency of the light engine, $L_{\text {source }}$ the luminance of the source, and $E_{\max }$ the maximum étendue limit of the system. If we assume that the source reaches the étendue limit of the system, then the screen brightness experienced by the user is directly linked to the luminance of the source. The luminance of the screen or screen brightness is given by

$$
L_{\text {screen }}=G_{\text {screen }} \frac{A_{\text {panel }}}{A_{\text {image }}} \mathrm{NA}_{\text {lens }}^{2} \eta L_{\text {source }} .
$$

For a given screen and image size, the screen brightness can, therefore, be increased in three ways.

- We can increase the étendue limit of the system $\left(A_{\text {panel }}\right.$ and $\mathrm{NA}_{\text {lens }}$ ).

- We can improve the efficiency of the light engine $(\eta)$.

- We can increase the luminance of the source $\left(L_{\text {source }}\right)$.

\section{INCREASING THE SYSTEM ÉTENDUE LIMIT}

Ideally, a system should be designed so that the system étendue is equal or larger that the étendue at the source. The larger the system étendue, the more light it will be able to collect. We can increase the system étendue limit by using a larger panel or a larger numerical aperture lens, and by insuring that no other components limit the beam propagation. This étendue increase will enable the use of more LEDs, or of a LED with a larger emissive area.

Let us consider a 0.85 -inch $(21.59 \mathrm{~mm})$ panel with a $4: 3$ aspect ratio and a lens with a numerical aperture equal to 0.2 $(\sim F / 2.5)$. The étendue limit is in this case $28.1 \mathrm{~mm}^{2} \cdot \mathrm{sr}$. In this example, we are using Luxeon Rebel LEDs from Lumileds. The die area is $1 \mathrm{~mm}^{2}$ and the refractive index of the encapsulant is about 1.5 . The emission pattern is very close to a lambertian emitter; therefore, the étendue of one Luxeon Rebel is approximately $7 \mathrm{~mm}^{2} \cdot \mathrm{sr}$. In this case, we are not étendue-limited as the étendue of the source is smaller than the étendue limit of the system. Therefore, we can use multiple LEDs in order to "fill" the available étendue. In this case, up to four LEDs can be arrayed. Adding more LEDs will not increase the lumen output of the projector, as we already reached the étendue limit. Arrays inevitably add extra space between the emissive areas, which can lead in some cases to an undesirable étendue increase and usually requires a complex architecture to achieve good spatial uniformity. For this reason, large size LED chips have been developed.

There are practical limitations to the system étendue in projectors though: the cost and complexity of the projection lens increases for low $f$-numbers, and polarization-dependant components such as polarizing beam splitters have strong angular dependencies that decrease contrast for low $f$-numbers. Microdisplays also have a limited size and angular acceptance. DMDs are designed for $\sim F / 2.4$ beams, even though an anamorphic $f$-number design $(F / 2.0 \times F / 2.4)$ has been investigated to increase the collected flux [3]. This design takes advantage of the fact that the beam angular constraint is tighter in the tilt direction of the micromirrors. In many applications compactness is a design constraint that limits the size of the components and therefore of the system étendue.

\section{DeCReasing Losses IN The Light Engine}

LED-based projectors can potentially be more efficient than lamp-based projectors. LEDs do not require IR, UV, and dichroic filters, and collection of light from LEDs is more efficient than from thermal light sources. However, recent designs of compact LED projectors report overall efficiencies between $3 \%$ and $8 \%$ [4], [5]. The total efficiency $\eta$ can be modeled as

$$
\eta=\eta_{C} \cdot \eta_{E} \cdot \eta_{I} \cdot \eta_{P} \cdot \eta_{\mu D} \cdot \eta_{C R} \cdot \eta_{L}
$$

where the following is defined.

- $\eta_{C}$ is the light collection efficiency. LEDs typically emit in a hemisphere, so collecting the entire flux with a regular condenser lens is unpractical. Nonetheless, LEDs can 


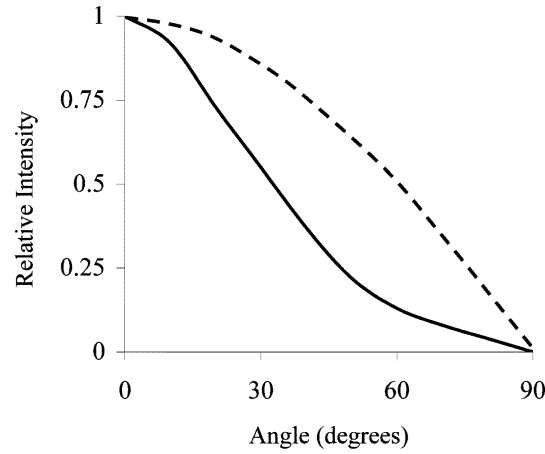

(a)

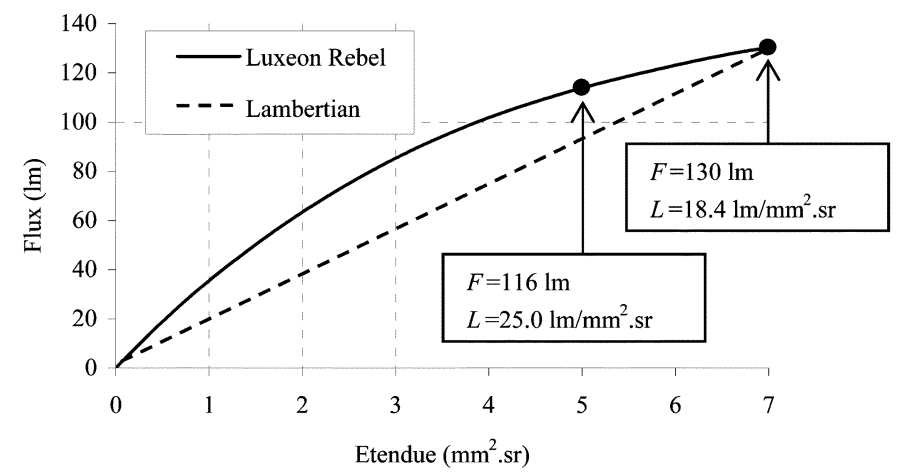

(b)

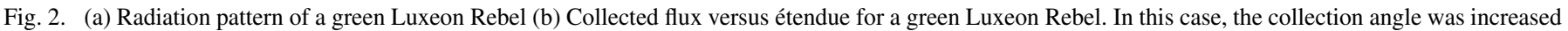

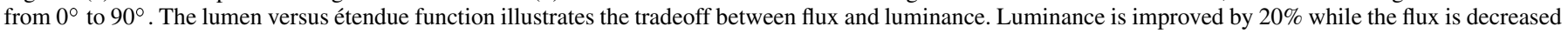
by $10 \%$.

be directly coupled to collection devices such as compound parabolic concentrators, tapered rods, or TIR lenses. These devices have already been widely investigated [2], [6] but new optimization techniques and manufacturing capabilities now allow the use of free-form shapes, thus increasing device efficiency and compactness [7]. A major problem is the coupling between the LED and the collector input face. Encapsulants and lenses enable higher extraction efficiencies but they increase the source étendue by a factor $n^{2}$ and prevent a direct coupling between the LED die and the collector, which also causes étendue increase (often referred as étendue dilution). For this reason, flat surface emitters that are easier to couple have been developed. These devices use alternative extraction improvement techniques, such as micro-reflector (Osram "Ostar", [8], [9]) and photonic lattices (Luminus "PhlatLight," [10], [11]).

- $\eta_{E}$ is the ratio between the system étendue limit and the étendue at the source. To ensure that no light is wasted, the étendue at the source should be equal or smaller than the system étendue limit.

- $\eta_{I}$ is the integrator efficiency. The integrator shapes the light beam and makes it uniform over the microdisplay. Two devices are most commonly used: lenslet arrays ("fly's eye") and mixing rods [12].

- $\eta_{P}$ is the polarization management efficiency (for liquid crystal devices), including polarizers and polarization conversion systems. This will be detailed in the last section. A thorough review of polarization technologies for LCD projection can be found in [13].

- $\eta_{\mu D}$ is the microdisplay efficiency. Typically reflective devices, such as LCoS and DMDs, have a higher efficiency than transmissive LCD, but their integration is more complex as they require a beam splitter to separate the incoming from the outgoing light. A thorough review of microdisplay technologies can be found in [14].

- $\eta_{C R}$ is the color recombination and color balance efficiency.

- $\eta_{L}$ is the projection lens efficiency.

Some additional general rules help making an optical system efficient. First, Fresnel losses occurring at each interface can be reduced by merging the components altogether. The con- centration and integration functions can be done by one single component such as tapered light pipes. Gradually tapered light pipes and lensed light pipes have been investigated to achieve better compactness [15], [16]. Polarizers, filters, and microdisplays can be glued to other components [4], [17]. Antireflection coatings are useful to reduce Fresnel losses but their angular dependency can be an issue for beams with large angular extents. Second, designs using TIR should be preferred over metalized reflective components, as they ensure lower light loss. Indeed, in the visible range TIR can reflect $99.9 \%$ of the light, whereas a metal with a good reflectance reflect $95 \%$ of the light [18]. However, designs using TIR are sensitive to material absorption and scattering and require antireflection coatings or index-matching gels to minimize Fresnel losses. Third, only a custom design of the collection optics can achieve good efficiency while shaping the beam for the rest of the optical system. Each LED model requires a specific design, according to the size and shape of the LED die, and the pattern of emission.

\section{INCREASING LUMINANCE}

\section{A. Increasing the Source Luminance}

Manufacturers often emphasize the emitted flux, while luminance is the most critical parameter for étendue-limited systems. Current LED luminance levels strongly limit the brightness that can be achieved by LED projectors. High intensity discharge lamps typically have luminance levels over $1000 \mathrm{~lm} / \mathrm{mm}^{2} \cdot \mathrm{sr}$. As a comparison, the current brightest LEDs still have an average luminance below $100 \mathrm{~lm} / \mathrm{mm}^{2} \cdot$ sr. Two main factors limit the luminance of an LED at the chip level. First, not all electrons injected into the semiconductor material are converted to photons; the efficiency of this process is known as the internal quantum efficiency. Much research effort has been put to develop materials with higher internal quantum efficiencies, but green LEDs still tend to have low internal quantum efficiencies compared to blue and red LEDs. Also, internal quantum efficiency decreases with higher current densities and increased chip temperature. Driving LEDs at high currents does increase their luminance, but at the expense of efficiency and lifetime. Overheating is a limiting factor in this case, so thermal management and semiconductor material quality is critical to achieve 


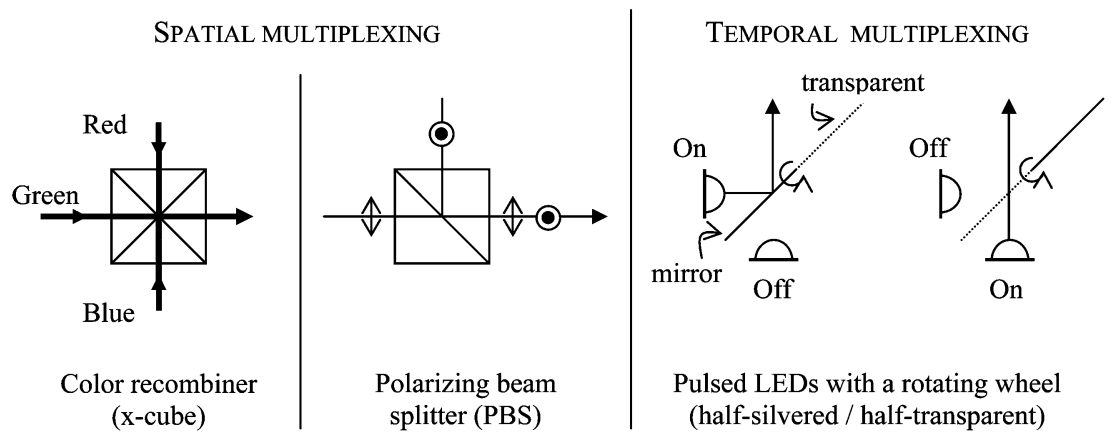

Fig. 3. Several LEDs can be multiplexed (i.e., combined within the same étendue) to get higher luminance. Spatial and temporal multiplexing are illustrated.

high luminance LED chips. Another important task is to provide an effective way of extracting light out of the light-emitting region. Semiconductor materials typically have high refractive indices, so more than half of the emitted light is trapped inside the chip because of total internal reflection. Several techniques have been investigated to counter this phenomenon, such as epoxies or silicon resins with refractive indices close to the chip refractive index, textured chip surfaces, shaped chips, microreflectors, and photonic lattices. In order to reduce light absorption in the electrodes and the substrate, alternative chip designs have been developed, such as thin films and flip chips [19].

Luminance can also be enhanced with the secondary optics, by taking advantage of nonuniformities in the source emission pattern. In this case, the secondary optics must restrain the beam to a smaller étendue where the average luminance is higher, as we shall detail next. The radiation pattern of most LEDs is not perfectly Lambertian. For this example we choose a LED model with a radiation pattern slightly peaked in the forward direction (green Luxeon Rebel). As a result, the collected flux versus étendue function is nonlinear (Fig. 2). Let's imagine we use a compound parabolic concentrator (CPC) directly coupled to the LED die to collect the light. Apart from Fresnel losses, a CPC will collect all the emitted light as it covers the whole emissive area and performs full angular collection. In this case the collected flux is about $130 \mathrm{~lm}$ and the average luminance $18.4 \mathrm{~lm} / \mathrm{mm}^{2}$. sr. If we use a simple lens instead of a CPC, light collection will be limited to a smaller angle. By collecting light only up to $60^{\circ}$, the collected flux decreased by $10 \%(116 \mathrm{~lm})$ while the average luminance increases by $20 \%\left(25 \mathrm{~lm} / \mathrm{mm}^{2} \cdot \mathrm{sr}\right)$. This illustrates a general tradeoff between collected flux and luminance: luminance can only be increased at the expense of the total flux. This analysis is not limited to the collection angle. The spatial and angular extents of a light source are never clearly bounded so it is critical to find a good compromise between flux and luminance. Lumen versus étendue functions such as the one shown in Fig. 2 are helpful to choose the optimal tradeoff [20]. A similar analysis with luminance versus flux functions was also carried on in [21].

Lasers can be seen as the ultimate high luminance sources, as their emitted flux is concentrated within a low étendue beam. Laser projectors are, therefore, not limited by luminance, but by the luminous flux of the laser source. Most laser projectors raster scan or line scan the image, so their architecture differs greatly from lamp-based and LED-based projectors. Image scanning can potentially lead to high image resolution. However, the high spatial and temporal coherence of laser light creates speckle patterns that can in some cases severely degrade the image quality, thus requiring the use of speckle reduction techniques [22]-[24].

\section{B. Multiplexing}

Luminance can be increased by combining multiple LEDs within the same étendue (spatial multiplexing). For instance, beams with nonoverlapping spectra can be combined. In projectors, the red, green and blue channels are usually combined with color recombiners known as $x$-cubes. More than three primary colors can be used, thus increasing luminance at the expense of a more complex color management [25], [26]. Beams with orthogonal polarizations can be combined with polarization beam splitters (PBS). LEDs can advantageously be pulsed, i.e., they can be driven at a high current during a short time. At constant power LEDs become slightly less efficient for higher currents and shorter "on" time, but we can take advantage of the "off" time to pulse other LEDs within the same étendue. Temporal multiplexing is usually achieved by synchronizing multiple LEDs with a rotating mirror so that only the on-state LEDs are coupled to the system [27], [28]. An alternative design involving no moving parts uses a PBS and a switchable half wave retarder [27] (see Fig. 3).

\section{Light Recycling}

Luminance can also be increased by "recycling light". When the source doesn't fully absorb its own radiation, light can be redirected to the source and then reflected by it within the same étendue. Reflected light is added to the light emitted by the source, therefore increasing its apparent luminance. Thus, the more reflective the source is and the more efficient light recycling will be. This principle works well with solid-state lighting [29] and can be implemented in various ways. All the devices follow the same technique which consists in implementing a reflecting cavity with an output étendue smaller than the source étendue, as illustrated on Fig. 4. In some designs the output area is made smaller (Goldeneye, [30]), in other designs the output beam divergence is constrained with prismatic structures using Total Internal Reflection (BEF film from 3M, [31]) or with microlenses [28]. Sometimes, light recycling is combined with the collection function [4] or with the polarization conversion function [32]. Reported efficiency gain with such systems ranges from 1.1 to 1.3 . 
(a)

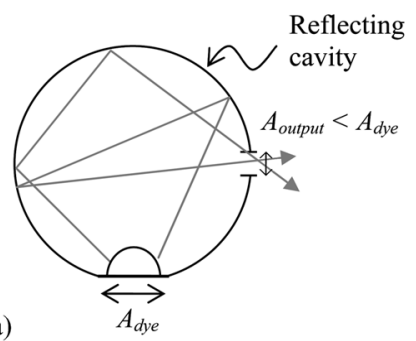

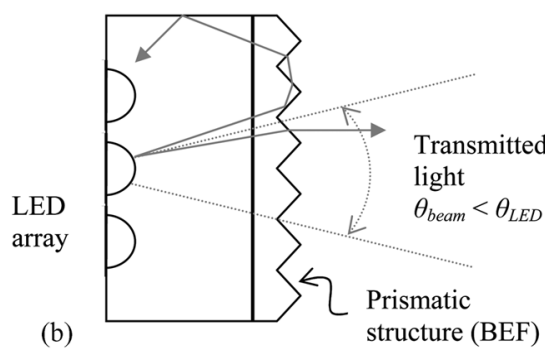

Fig. 4. Light recycling systems where étendue is reduced by (a) limiting the output area or (b) limiting the angular extent of the beam. Luminance is increased at the expense of the flux. The efficiency of the recycling process strongly depends on the reflectivity of the cavity walls and of the LED die.

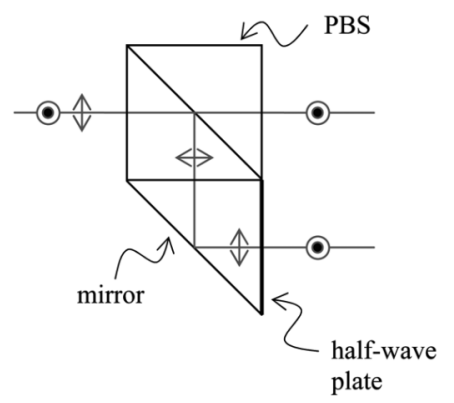

(a)

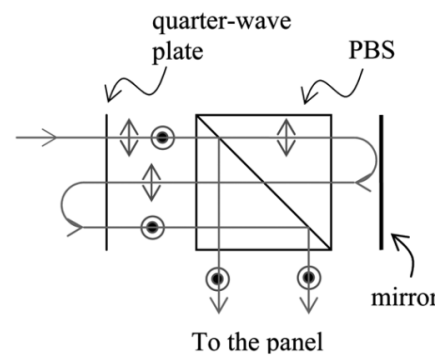

(b)
Fig. 5. (a) Polarization conversion system which doubles the étendue of the beam and (b) polarization recycling system where étendue is conserved. Polarization recycling efficiency depends on the reflectance of the light source.

\section{POlarization CONVERSION}

$\mathrm{LCoS}$ and transmissive LCD panels require linearly polarized light. As the light emitted by LED sources is randomly polarized, only $50 \%$ of the light has the required polarization direction. Polarization conversion systems have been developed to avoid wasting the remaining $50 \%$ of light with the "wrong" polarization. The most common polarization conversion system is a combination of a polarizing beam splitter (PBS), a mirror and a half-wave plate as shown in Fig. 5. It is sometimes used in the form of an array [20]. This type of PCS can reach up to $85 \%$ transmission with the required polarization, but it doubles the étendue of the system (beam extent is doubled). For étenduelimited systems, doubling the étendue means losing $50 \%$ of the light. The overall transmission of the system is then $0.5 \times$ $0.85=42.5 \%$, which is roughly equal to the transmission of a good polarizer. Therefore, polarization conversion systems which double the étendue have no advantage for étendue-limited systems.

An alternative for étendue-limited systems is to recycle the light with the wrong polarization: light with the correct polarization is transmitted, whereas light with the wrong polarization is reflected back towards the LED. This principle has been implemented with a MacNeille prism [32] and with a reflective polarizer. A quarter-wave plate is in theory required to convert the recycled polarization. Similar results were obtained without it, which can be explained by the polarization being randomized through multiple reflections [4]. If plastic components are used, their birefringence may also be responsible for changes in the polarization state. A $29 \%$ gain in transmission was obtained this way, without increasing the étendue. Another solution is to halve the étendue with classical light recycling, and then to use a standard polarization conversion system which would double the étendue [5].

\section{CONCLUSION}

Obtaining high brightness LED projection displays requires a comprehensive system design approach. The light engine needs to be designed in order to match the characteristics of the source, the microdisplay and the projection lens. New high brightness LEDs with surface emission have been designed to fit the needs of projection displays. Improvement in solid-state technologies is also expected to improve the die reflectivity and thus make light recycling more efficient in the future. However, improvement in projector brightness does not entirely rely on advances in LED technologies. Recent progress in manufacturing capabilities and optimization techniques for nonimaging optics enables custom design components that can take into account inhomogeneities in the LED spatial and angular emission patterns, thus approaching the maximum theoretical efficiency given by the étendue conservation law. Even though brightness is a key parameter in the device performance, the optimization process should also be driven by specific metrics taking into account the performance goals, including contrast, image uniformity, system size, and cost requirements.

\section{ACKNOWLEDGMENT}

The authors thank VDC Display Systems and the Office of Naval Research (ONR) Virtual Technologies and Environments (VIRTE) program for motivating this review and for their support. They thank Optical Research Associates for the educational license of LightTools ${ }^{\circledR}$ that allowed them, through simulations, to deepen their understanding of many of the concepts described in this paper. Finally, the authors thank W. J. Cassarly for insightful conversations about illumination designs.

\section{REFERENCES}

[1] C. DeCusatis, Handbook of Applied Photometry. Woodbury, NY: AIP Press, 1997.

[2] R. Winston, J. C. Minano, P. Benitez, and W. T. Welford, Nonimaging Optics. Amsterdam, The Netherlands: Elsevier Academic, 2005.

[3] C.-M. Cheng and J.-L. Chern, "Design of a dual-f-number illumination system and its application to DMD ${ }^{\mathrm{TM}}$ projection displays," J. Soc. Inf. Display, vol. 14, pp. 819-827, 2006.

[4] M. P. Krijn, B. A. Salters, and O. H. Willemsen, "LED-based miniprojectors," in Photonics in Multimedia, Strasbourg, France, 2006.

[5] W. Y. Lee, Y. C. Lee, K. Sokolov, H. J. Lee, and I. Moon, "LED projection displays," in Nonimaging Optics and Efficient Illumination Systems, Denver, CO, 2004 
[6] W. J. Cassarly, "Nonimaging optics: Concentration and illumination," in Handbook of Optics, 2nd ed. New York: McGraw-Hill, 1995, vol. 3 , ch. 2.

[7] T. L. R. Davenport, T. A. Hough, and W. J. Cassarly, "Optimization for illumination systems: The next level of design," in Photon Management, Strasbourg, France, 2004.

[8] K. Gerhard, G. Stefan, B. Nicole, S. Wolfgang, and W. Stefan, "58.3: A new LED light source for projection applications," in SID Symp. Dig. Tech. Papers, 2005, vol. 36, pp. 1702-1705.

[9] N. Breidenassel, S. Grotsch, and W. Schnabel, "61.3: LED light source for RPTV applications," in SID Symp. Dig. Tech. Papers, 2006, vol. 37, pp. 1816-1818.

[10] A. A. Erchak, D. J. Ripin, S. Fan, P. Rakich, J. D. Joannopoulos, E. P. Ippen, G. S. Petrich, and L. A. Kolodziejski, "Enhanced coupling to vertical radiation using a two-dimensional photonic crystal in a semiconductor light-emitting diode," Appl. Phys. Lett., vol. 78, pp. 563-565, 2001.

[11] C. Hoepfner, "61.1: Invited paper: PhlatLight ${ }^{\mathrm{TM}}$ photonic lattice LEDs for RPTV light engines," in SID Symp. Tech. Papers, 2006, vol. 37, pp. $1808-1811$.

[12] B. Van Giel, Y. Meuret, and H. Thienpont, "Using a fly's eye integrator in efficient illumination engines with multiple light-emitting diode light sources," Opt. Eng., vol. 46, pp. 043001-6, 2007.

[13] M. G. Robinson, J. Chen, and G. D. Sharp, Polarization Engineering for LCD Projection. Hoboken, NJ: Wiley, 2005.

[14] D. Armitage, I. Underwood, and S.-T Wu, Introduction to Microdisplays. Hoboken, NJ: Wiley, 2006.

[15] H. Murat, D. Cuypers, and H. De Smet, "Design of new collection systems for multi LED light engines," in Photonics in Multimedia, Strasbourg, France, 2006.

[16] K. K. Li, S. Inatsugu, and S. Sillyman, "Design and optimization of tapered light pipes," in Nonimaging Optics and Efficient Illumination Systems, Denver, CO, 2004

[17] J. M. Teijido, F. Ludley, O. Ripoll, M. Ueda, and Y. Oshima, "73.2: Compact three panel LED projector engine for portable applications," in SID Symp. Dig. Tech. Papers, 2006.

[18] L. A. Whitehead and M. A. Mossman, "Off the beaten path with total internal reflection," presented at the 2006 Int. Opt. Des. Conf., Bellingham, WA.

[19] M. R. Krames, O. B. Shchekin, R. Mueller-Mach, G. O. Mueller, Z. Ling, G. Harbers, and M. G. Craford, "Status and future of high-power light-emitting diodes for solid-state lighting," J. Display Technol., vol. 3 , no. 2, pp. 160-175, Jun. 2007.

[20] E. H. Stupp and M. S. Brennesholtz, Projection Displays. New York: Wiley, 1999.

[21] J. Muschaweck and H. Ries, "Characterization of the thermodynamic quality of light sources," in Nonimaging Optics and Efficient Illumination Systems II, San Diego, CA, 2005.

[22] R. Martinsen, K. Kennedy, and A. Radl, "Speckle in laser imagery: Efficient methods of quantification and minimization," presented at the 1999 IEEE Lasers and Electro-Optics Society 12th Annu. Meeting (LEOS '99), San Francisco, CA, Nov. 8-11, 1999.

[23] I. T. Jahja, "Speckle contrast reduction in laser projection displays," in Proc. SPIE Int. Soc. Opt. Eng. 4657, 2002, vol. 131.

[24] J. W. Goodman, Speckle Phenomena in Optics: Theory and Applications. Englewood, CO: Roberts \& Co, 2007.

[25] T. Ajito, T. Obi, M. Yamaguchi, and N. Ohyama, "Expanded color gamut reproduced by six-primary projection display," in Projection Displays 2000: Sixth in a Series, San Jose, CA, 2000.

[26] S. Roth and W. Caldwell, "64.4: Four primary color projection display," in SID Symp. Dig. Tech. Papers, 2005, vol. 36, pp. 1818-1821.
[27] H. Murat, H. De Smet, D. Cuypers, Y. Meuret, H. Thienpont, M. Vervaeke, and L. Desmet, "Increased lumens per etendue by combining pulsed LEDs," in Projection Displays XI, San Jose, CA, 2005.

[28] J. Chaves, W. Falicoff, P. Benitez, J. C. Minano, W. A. Parkyn, and O. Dross, "Luminance enhancement of LED light sources for etenduelimited applications," in Nonimaging Optics and Efficient Illumination Systems III, San Diego, CA, 2006.

[29] L. Fu, R. Leutz, and H. Ries, "Light recycling in solid state devices," in Fifth Int. Conf. Solid State Lighting, San Diego, CA, 2005.

[30] K. Beeson, S. Zimmerman, W. Livesay, R. Ross, C. Livesay, and K. Livesay, "61.5: LED-based light-recycling light sources for projection displays," in SID Symp. Dig. Tech. Papers, 2006, vol. 37, pp. 1823-1826.

[31] R. C. Allen, 3M Innovative Properties Company, St. Paul, MN, USA "Brightness enhancement film," U.S. Patent 6760 157, Jul. 6, 2004.

[32] Y. Wang, "Efficiency enhancement of liquid crystal projection displays using light recycle technology," in Projection Displays VIII, San Jose, CA, 2002.

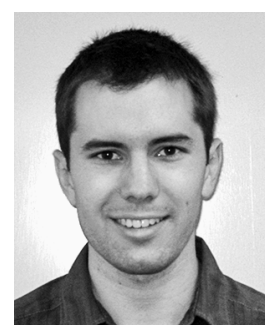

Florian Fournier (S'07) was born in Montpellier, France. He received the B.S. and M.S. degrees in optics from the Institut d'Optique in Orsay, France, in 2003 and 2005, respectively. He is currently working toward the Ph.D. degree in optical science from the University of Central Florida (UCF), Orlando, with Dr J. Rolland. His focus is on nonimaging optics and illumination design related to projection displays.

$\mathrm{He}$ has held an internship position or worked for Optical Research Associates, Pasadena, CA; VDC Display Systems, Cape Canaveral, FL; MSC Inspection Worldwide, Lyon, France; the French Museum Research Center (C2RMF) in Paris, France.

Mr Fournier is a student member of the Optical Society of America, SPIE and Society of Information Display (SID). He was the recipient of the 2007 Michael Kidger Memorial Scholarship.

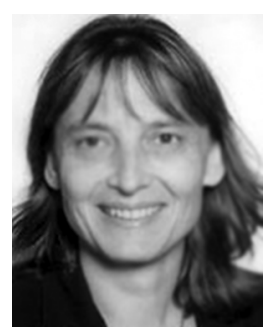

Jannick Rolland (M'96) was born in Africa and raised in Europe. She received a Diploma from the Institut D'Optique, Graduate School in France in 1984, and the Ph.D. degree in optical science from the University of Arizona, Tucson, in 1990.

She is a Professor of Optics at the University of Central Florida with joint appointments in Computer Science and Modeling and Simulation. After completing a Postdoctoral Fellowship, she joined the Research Faculty at UNC-Chapel Hill in 1992 and headed the Vision Research Group 1992-1996. She holds 13 patents, wrote 6 book chapters, and has over 60 peered review publications related to optical design, augmented reality, vision, and image quality assessment.

Professor Rolland served on the editorial board of the Journal Presence (MIT Press, 1996-2006), and as Associate Editor of Optical Engineering (1999-2004). She is a Guest Editor for a Special Issue on Medical Displays in an upcoming issue of the IEEE JOURNAL OF DisPlay TECHNOLOGY. She is a Fellow of the Optical Society of America, and a member of SPIE, and of Society of Information Display (SID). 